\title{
ACCURATE SOLUTION ESTIMATES FOR NONLINEAR NONAUTONOMOUS VECTOR DIFFERENCE EQUATIONS
}

RIGOBERTO MEDINA AND M. I. GIL'

Received 27 August 2002

The paper deals with the vector discrete dynamical system $x_{k+1}=A_{k} x_{k}+f_{k}\left(x_{k}\right)$. The wellknown result by Perron states that this system is asymptotically stable if $A_{k} \equiv A=$ const is stable and $f_{k}(x) \equiv \tilde{f}(x)=o(\|x\|)$. Perron's result gives no information about the size of the region of asymptotic stability and norms of solutions. In this paper, accurate estimates for the norms of solutions are derived. They give us stability conditions for (1.1) and bounds for the region of attraction of the stationary solution. Our approach is based on the "freezing" method for difference equations and on recent estimates for the powers of a constant matrix. We also discuss applications of our main result to partial reactiondiffusion difference equations.

\section{Introduction and notation}

Let $\mathbb{C}^{n}$ be the set of $n$-complex vectors endowed with the Euclidean norm $\|\cdot\|$. Consider in $\mathbb{C}^{n}$ the equation

$$
x_{k+1}=A_{k} x_{k}+f_{k}\left(x_{k}\right) \quad(k=0,1,2, \ldots)
$$

where $A_{k}(k=0,1,2, \ldots)$ are $n \times n$-complex matrices and $f_{k}: \mathbb{C}^{n} \rightarrow \mathbb{C}^{n}$ are given functions. A well-known result of Perron which dates back to 1929 (see [11, page 270], [8, Theorem 9.14], and [6]) states that (1.1) is asymptotically stable if $A_{k} \equiv A=$ const is stable (i.e., the spectral radius $r_{s}(A)$ of $A$ is less than 1) and $f_{k}(x)=\tilde{f}(x)=o(\|x\|)$. Clearly, this result is purely local. It gives no information about the size of the region of asymptotic stability and norms of solutions.

In this paper, we derive accurate estimates for the norms of solutions. Our approach is based on the "freezing" method for difference equations and on recent estimates for the powers of a constant matrix. Note that the "freezing" method for difference equations was developed in [5]. It is based on the relevant ideas for differential equations (cf. [2, 3, 7]).

Firstly, we consider the linear difference equation

$$
x_{k+1}=A_{k} x_{k} \quad(k=0,1,2, \ldots) .
$$


As it is well known, the fundamental matrix $X(k)$ of (1.2) can be expressed as

$$
X(m)=A_{m} A_{m-1} \cdots A_{0}
$$

But such a representation does not yield much information about the asymptotic value of solutions, except in the case of constant coefficients $A_{k}=A(k=0,1,2, \ldots)$, when $X(k)=A^{k}$ and the Jordan canonical form of $A$ determines the asymptotic behavior of the solutions. To prove the stability of (1.2) is equivalent to proving the boundedness of the sequence $\{\|X(m)\|\}_{1}^{\infty}$. This problem is easy to solve under the condition $\sup _{k}\left\|A_{k}\right\| \leq 1$. But it is rather restrictive. The "freezing" method allows us to avoid this condition in the case

$$
\left\|A_{k}-A_{j}\right\| \leq q_{k-j} \quad\left(q_{k}=q_{-k}=\mathrm{const} \geq 0, q_{0}=0 ; j, k=0,1,2, \ldots\right) .
$$

For example, if $A_{k}=\sin (c k) B(c=$ const $>0)$, where $B$ is a constant matrix, then condition (1.4) holds with $q_{k}=2\|B\||\sin (c k / 2)|$, since

$$
\sin \alpha-\sin \beta=2 \sin \left(\frac{\alpha-\beta}{2}\right) \cos \left(\frac{\alpha+\beta}{2}\right)
$$

for real $\alpha, \beta$.

For an $n \times n$-matrix $A$, denote

$$
g(A)=\left[N^{2}(A)-\sum_{j=1}^{n}\left|\lambda_{j}(A)\right|^{2}\right]^{1 / 2},
$$

where $N(A)$ is the Frobenius (Hilbert-Schmidt) norm of a matrix $A: N^{2}(A)=$ $\operatorname{Trace}\left(A A^{*}\right)$, and $\lambda_{1}(A), \lambda_{2}(A), \ldots, \lambda_{n}(A)$ are the eigenvalues of $A$ including their multiplicities. The relations

$$
\begin{gathered}
g(A) \leq\left[N^{2}(A)-\left|\operatorname{Trace}\left(A^{2}\right)\right|\right]^{1 / 2}, \\
g(A) \leq \sqrt{\frac{1}{2}} N\left(A^{*}-A\right)
\end{gathered}
$$

are true [3, Section 2.1]. Here $A^{*}$ is the adjoint matrix. If $A$ is a normal matrix: $A^{*} A=$ $A A^{*}$, then $g(A)=0$. If $A=\left(a_{i j}\right)$ is a triangular matrix such that $a_{i j}=0$ for $1 \leq j \leq i \leq n$, then

$$
g^{2}(A)=\sum_{1 \leq i \leq j \leq n}\left|a_{i j}\right|^{2} .
$$

For a natural number $n>1$, introduce the numbers

$$
\gamma_{n, p}=\sqrt{\frac{\mathbb{C}_{n-1}^{p}}{(n-1)^{p}}}
$$


for $p=1,2, \ldots, n-1$ and $\gamma_{n, 0}=1$. Here and below,

$$
\mathbb{C}_{m}^{k}=\frac{m !}{(m-k) ! k !} \quad(k=0,1,2, \ldots, m ; m=1,2, \ldots)
$$

are the binomial coefficients. Evidently, for $n>2$,

$$
\gamma_{n, p}^{2}=\frac{(n-2)(n-3) \cdots(n-p)}{(n-1)^{p-1} p !} \leq \frac{1}{p !} .
$$

Due to [4, Theorem 1.2.1], for any $n \times n$-matrix $A$, the inequality

$$
\begin{aligned}
\left\|A^{m}\right\| & \leq \sum_{k=0}^{m_{1}} \frac{m ! r_{s}^{m-k}(A) g^{k}(A) \gamma_{n, k}}{(m-k) ! k !} \\
& =\sum_{k=0}^{m_{1}} \mathbb{C}_{m}^{k} r_{s}^{m-k}(A) g^{k}(A) \gamma_{n, k} \quad\left(m_{1}=\min \{n-1, m\}\right)
\end{aligned}
$$

holds for every integer $m$, where $r_{s}(A)$ is the spectral radius of $A$.

\section{Preliminary facts}

Firstly, we recall a boundedness result for (1.2) which is proven in [5, Lemma 1.1], namely, we recall the following lemma.

LeMma 2.1. Under condition (1.4), let

$$
\zeta_{0} \equiv \sum_{k=1}^{\infty} q_{k} \sup _{l=1,2, \ldots}\left\|A_{l}^{k}\right\|<1 .
$$

Then, every solution $\left\{x_{k}\right\}$ of (1.2) satisfies the inequality

$$
\sup _{k=1,2, \ldots}\left\|x_{k}\right\| \leq \beta_{0}\left\|x_{0}\right\|\left(1-\zeta_{0}\right)^{-1},
$$

where

$$
\beta_{0}=\sup _{k, l=0,1,2, \ldots}\left\|A_{l}^{k}\right\| .
$$

As a consequence, it is possible to establish the next corollaries.

Corollary 2.2. Let the conditions

$$
\begin{aligned}
\left\|A_{k}-A_{k+1}\right\| & \leq \tilde{q} \quad(k=1,2, \ldots ; \tilde{q}=\text { const }>0), \\
\theta_{0} & \equiv \sum_{k=1}^{\infty} \sup _{l=1,2, \ldots}\left\|A_{l}^{k}\right\| k<\tilde{q}^{-1}
\end{aligned}
$$

hold. Then, every solution $\left\{x_{k}\right\}$ of (1.2) satisfies the inequality

$$
\left\|x_{k}\right\| \leq \beta_{0}\left\|x_{0}\right\|\left(1-\tilde{q} \theta_{0}\right)^{-1} \quad(k=1,2, \ldots) .
$$


606 Solution estimates for difference equations

Indeed, under condition (2.4), we have

$$
\left\|A_{k}-A_{j}\right\| \leq \tilde{q}|k-j| \quad(j, k=0,1,2, \ldots)
$$

So $\zeta_{0} \leq \tilde{q} \theta_{0}$. Now, the required result follows from Lemma 2.1.

Corollary 2.3. Let condition (2.4) hold. In addition, for a constant $v>0$, let

$$
\theta(v) \equiv \sum_{k=1}^{\infty} v^{-k-1} \sup _{l=1,2, \ldots}\left\|A_{l}^{k}\right\| k<\tilde{q}^{-1} .
$$

Then, every solution $\left\{x_{k}\right\}$ of (1.2) satisfies the inequality

$$
\left\|x_{k}\right\| \leq v^{k} m(v)\left\|x_{0}\right\|(1-\tilde{q} \theta(v))^{-1} \quad(k=1,2, \ldots),
$$

where

$$
m(v) \equiv \sup _{l, k=0,1,2, \ldots} v^{-k}\left\|A_{l}^{k}\right\|
$$

Indeed, due to condition (2.8), $m(v)<\infty$. Putting $x_{k}=v^{k} z_{k}$ in $(1.2)$, we get

$$
z_{k+1}=v^{-1} A_{k} z_{k}
$$

Corollary 2.2 and condition (2.8) imply

$$
\sup _{k=1,2, \ldots}\left\|z_{k}\right\| \leq m(v)\left\|z_{0}\right\|(1-\tilde{q} \theta(v))^{-1} \quad(k=1,2, \ldots) .
$$

Hence, the required estimate follows. Recall also the following result from [5].

Theorem 2.4. Under condition (1.4), let

$$
\begin{gathered}
\rho_{0} \equiv \sup _{l=1,2, \ldots} r_{s}\left(A_{l}\right)<1, \quad v_{0} \equiv \sup _{l=0,1,2, \ldots} g\left(A_{l}\right)<\infty, \\
\xi \equiv \sum_{m=1}^{\infty} \sum_{k=0}^{n-1} \mathbb{C}_{m}^{k} \rho_{0}^{m-k} v_{0}^{k} \gamma_{n, k} q_{m}<1 .
\end{gathered}
$$

Then, every solution $\left\{x_{k}\right\}$ of (1.2) is bounded. Moreover,

$$
\sup _{k=1,2, \ldots}\left\|x_{k}\right\| \leq M_{0}\left\|x_{0}\right\|(1-\xi)^{-1},
$$

where

$$
M_{0}=\sum_{k=0}^{n-1} v_{0}^{k} \gamma_{n, k}\left(\psi_{k}+k\right)^{k} \rho_{0}^{\psi_{k}}
$$

with $\psi_{k}=\max \left\{0,-k\left(1+\log \rho_{0}\right) / \log \rho_{0}\right\}$. 


\section{The main result}

The previous estimates give us a possibility to investigate (1.1) as a nonlinear perturbation of (1.2). For a positive $r \leq \infty$, denote the ball

$$
B_{r}=\left\{x \in \mathbb{C}^{n}:\|x\| \leq r\right\}
$$

and assume that there are constants $\mu, \nu \geq 0$, such that

$$
\left\|f_{k}(x)\right\| \leq \nu\|x\|+\mu \quad\left(x \in B_{r} ; k=0,1,2, \ldots\right) .
$$

Recall that the quantities $\rho_{0}, v_{0}$, and $M_{0}$ are defined by (2.13) and (2.16). Let

$$
\psi(A) \equiv \sum_{k=0}^{\infty} \sum_{j=0}^{n-1} \mathbb{C}_{k}^{j} \rho_{0}^{k-j} v_{0}^{j} \gamma_{n, j}
$$

Now we are in a position to formulate the main result of the paper.

Theorem 3.1. Under the conditions (1.4), (2.13), and (3.2), let

$$
S(f ; A) \equiv \sum_{k=0}^{\infty} \sum_{j=0}^{n-1} \mathbb{C}_{k}^{j} \rho_{0}^{k-j} v_{0}^{j} \gamma_{n, j}\left(q_{k}+\nu\right)<1 .
$$

Then, any solution $\left\{x_{k}\right\}_{=0}^{\infty}$ of (1.1) satisfies the inequality

$$
\sup _{k=1,2, \ldots}\left\|x_{k}\right\| \leq \frac{M_{0}\left\|x_{0}\right\|+\mu \psi(A)}{1-S(f ; A)},
$$

provided that

$$
\frac{M_{0}\left\|x_{0}\right\|+\mu \psi(A)}{1-S(f ; A)} \leq r
$$

The proof of this theorem is given afterwards.

Recall that

$$
\beta_{0}=\sup _{k, l=0,1, \ldots}\left\|A_{l}^{k}\right\|
$$

and let

$$
\theta_{1} \equiv \sum_{k=0}^{\infty} \sup _{l=0,1, \ldots}\left\|A_{l}^{k}\right\|
$$

Lemma 3.2. Under conditions (1.4) and (3.2), let

$$
S_{0} \equiv \sum_{k=0}^{\infty}\left(q_{k}+v\right) \sup _{l=0,1,2, \ldots}\left\|A_{l}^{k}\right\|<1 .
$$


608

Solution estimates for difference equations

Then, every solution $\left\{x_{k}\right\}$ of (1.1) satisfies the inequality

$$
\left\|x_{k}\right\| \leq\left[\beta_{0}\left\|x_{0}\right\|+\theta_{1} \mu\right]\left(1-S_{0}\right)^{-1} \quad(k=1,2, \ldots),
$$

provided that

$$
\left[\beta_{0}|| x_{0} \|+\theta_{1} \mu\right]\left(1-S_{0}\right)^{-1} \leq r .
$$

Proof. Rewrite (1.1) as

$$
x_{k+1}-A_{l} x_{k}=\left(A_{k}-A_{l}\right) x_{k}+f_{k}\left(x_{k}\right)
$$

with a fixed integer $l$. The variation of parameters formula yields

$$
x_{l+1}=A_{l}^{l+1} x_{0}+\sum_{j=0}^{l} A_{l}^{l-j}\left[\left(A_{j}-A_{l}\right) x_{j}+f_{j}\left(x_{j}\right)\right] .
$$

There are two cases to consider: $r=\infty$ and $r<\infty$. First, assume that (3.2) is valid with $r=\infty$, then, by (1.4),

$$
\begin{aligned}
\left\|x_{l+1}\right\| & \leq \beta_{0}\left\|x_{0}\right\|+\sum_{j=0}^{l}\left\|A_{l}^{l-j}\right\|\left[q_{l-j}\left\|x_{j}\right\|+\nu\left\|x_{j}\right\|+\mu\right] \\
& \leq \beta_{0}\left\|x_{0}\right\|+\sum_{j=0}^{l}\left\|A_{l}^{l-j}\right\|\left(q_{l-j}+\nu\right)\left\|x_{j}\right\|+\theta_{1} \mu \\
& \leq \beta_{0}\left\|x_{0}\right\|+\max _{k=0, \ldots, l}\left\|x_{k}\right\| \sum_{k=0}^{l}\left\|A_{l}^{k}\right\|\left(q_{k}+\nu\right)+\mu \theta_{1} \\
& \leq \beta_{0}\left\|x_{0}\right\|+\max _{k=1, \ldots, l}\left\|x_{k}\right\|\left(\sum_{k=0}^{\infty}\left(q_{k}+\nu\right) \sup _{l=0,1,2, \ldots}\left\|A_{l}^{k}\right\|\right)+\mu \theta_{1} .
\end{aligned}
$$

Consequently,

$$
\max _{k=1,2, \ldots, l+1}\left\|x_{k}\right\| \leq \beta_{0}\left\|x_{0}\right\|+S_{0} \max _{k=0,1, \ldots, l+1}\left\|x_{k}\right\|+\mu \theta_{1}
$$

But $\beta_{0} \geq 1$. So

$$
\max _{k=0,1,2, \ldots, l+1}\left\|x_{k}\right\| \leq \beta_{0}\left\|x_{0}\right\|+S_{0} \max _{k=0,1, \ldots, l+1}\left\|x_{k}\right\|+\mu \theta_{1} .
$$

Hence,

$$
\sup _{k=0,1,2, \ldots}\left\|x_{k}\right\| \leq \frac{\beta_{0}\left\|x_{0}\right\|+\mu \theta_{1}}{1-S_{0}} .
$$

Let now $r<\infty$. Define the function

$$
\tilde{f}_{k}(x)= \begin{cases}f_{k}(x), & \|x\| \leq r \\ 0, & \|x\|>r .\end{cases}
$$


Since

$$
\left\|\tilde{f}_{k}(x)\right\| \leq \nu\|x\|+\mu, \quad k=0,1, \ldots ; x \in B_{\infty},
$$

then the sequence $\left\{\widetilde{x}_{k}\right\}_{=0}^{\infty}$ defined by

$$
\widetilde{x_{0}}=x_{0}, \quad \widetilde{x_{k+1}}=A_{k} \widetilde{x_{k}}+\tilde{f}_{k}\left(\widetilde{x_{k}}\right), \quad k=0,1, \ldots,
$$

satisfies the inequality

$$
\sup _{k=0,1, \ldots}\left\|\widetilde{x_{k}}\right\| \leq \frac{\beta_{0}\left\|x_{0}\right\|+\mu \theta_{1}}{1-S_{0}} \leq r
$$

according to the above arguments and condition (3.11). But $f$ and $\tilde{f}_{k}(x)$ coincide on $B_{r}$. So $x_{k}=\widetilde{x_{k}}$ for $k=0,1,2, \ldots$. Therefore, $(3.10)$ is satisfied, thus concluding the proof.

proof of Theorem 3.1. As it was proved in [5, Lemma 2.2], $\beta_{0} \leq M_{0}$. Moreover, due to (1.12), we have $\theta_{1} \leq \psi(A)$ and $S_{0} \leq S(f: A)$. Now the result is due to Lemma 3.2.

Remarks 3.3. (a) Under (3.2) with $\mu=0, f_{k}(0)=0$ so that $\{0\}$ is a solution of (1.1). Under condition $S(f, A)<1$, Theorem 3.1 asserts that the trivial solution is stable, and that any initial vector $x_{0} \in B_{r}$, satisfying the condition

$$
\left\|x_{0}\right\| \leq \frac{(1-S(f, A)) r}{M_{0}}
$$

belongs to the region of attraction.

(b) If $v \equiv 0$, then every solution of (1.1) with the initial vector $x_{0}$ satisfying

$$
\left\|x_{0}\right\| M_{0}+\mu \psi(A) \leq(1-\xi) r
$$

is bounded.

\section{Applications}

In this section, we will illustrate our main results by considering a partial difference equation. We consider a simple three-level discrete reaction-diffusion equation of the form

$$
u_{i}^{(j+1)}=a_{j} u_{i-1}^{(j)}+b_{j} u_{i}^{(j)}+c_{j} u_{i+1}^{(j)}+g_{i}^{(j)}+f_{j}\left(u_{i}^{(j)}\right),
$$

defined on $\Omega=\{(i, j): i=0,1, \ldots, n+1 ; j=0,1, \ldots\}$, where $\left\{a_{j}\right\},\left\{b_{j}\right\}$, and $\left\{c_{j}\right\}$ are real sequences, $g=\left\{g_{i}^{(j)}\right\}$ is a complex function defined on $\Omega$, and $f_{j}: \mathbb{C} \rightarrow \mathbb{C}(j=0,1, \ldots)$ are given functions. Assume that the side conditions

$$
\begin{aligned}
u_{0}^{(j)}=\delta_{j} \in \mathbb{C}, & j=0,1, \ldots, \\
u_{n+1}^{(j)}=\gamma_{j} \in \mathbb{C}, & j=0,1, \ldots, \\
u_{i}^{(0)}=\tau_{j} \in \mathbb{C}, & i=1,2, \ldots, n,
\end{aligned}
$$


are imposed, where $\tau=\left(\tau_{1}, \tau_{2}, \ldots, \tau_{n}\right) \in \mathbb{C}^{n}$. A solution of problem (4.1), (4.2), (4.3), and (4.4) is a discrete function $u=\left\{u_{i}^{(j)}\right\}_{(i, j) \in \Omega}$ which satisfies relations (4.1), (4.2), (4.3), and (4.4). The existence and uniqueness of solutions to that problem is obvious, provided that $f_{j}$ is one-one valued. With the notation

$$
u^{(j)}=\left(u_{1}^{(j)}, u_{2}^{(j)}, \ldots, u_{n}^{(j)}\right)
$$

the sequence $\left\{u^{(j)}\right\}_{j=0}^{\infty}$ satisfies the vector equation

$$
u^{(j+1)}=A_{j} u^{(j)}+G_{j}+F_{j}\left(u^{(j)}\right), \quad j=0,1, \ldots,
$$

and the initial condition

$$
u^{(0)}=\tau,
$$

where

$$
\begin{aligned}
A_{j} & =\left[\begin{array}{cccccc}
b_{j} & c_{j} & 0 & \ldots & \ldots & 0 \\
a_{j} & b_{j} & c_{j} & 0 & \ldots & 0 \\
0 & a_{j} & b_{j} & c_{j} & \ldots & 0 \\
\vdots & \vdots & \vdots & \vdots & \vdots & \vdots \\
0 & \ldots & \ldots & 0 & a_{j} & b_{j}
\end{array}\right], \quad j=0,1,2, \ldots, \\
G_{j} & =\left(g_{1}^{(j)}, \ldots, g_{n}^{(j)}\right)+\left(a_{j} \delta_{j}, 0, \ldots, 0, c_{j} \gamma_{j}\right), \\
F_{j}(x) & =\left(f_{j}\left(x_{1}\right), \ldots, f_{j}\left(x_{n}\right)\right), \quad x=\left(x_{1}, x_{2}, \ldots, x_{n}\right) .
\end{aligned}
$$

Thus, we can write problem (4.1), (4.2), (4.3), and (4.4) as (1.1) with

$$
f_{j}(x)=F_{j}(x)+G_{j} .
$$

Assume that there are nonnegative constants $\mu_{1}$ and $\nu$ such that

$$
\left\|F_{j}(x)\right\| \leq \nu\|x\|+\mu_{1} \quad\left(x \in B_{r} ; j=1,2, \ldots\right) .
$$

In addition,

$$
\mu_{2} \equiv \sum_{j=0}^{\infty}\left\|G_{j}\right\|<\infty .
$$

So condition (3.2) holds with $\mu_{0}=\mu_{1}+\mu_{2}$. As a direct consequence of Theorem 3.1, we get the following theorem.

Theorem 4.1. Let conditions (1.4), (4.2), (4.10), and (4.11) hold with $\mu=\mu_{1}+\mu_{2}$ and $x_{0}=\tau$. Then, the unique solution $x_{j}=\left\{u_{i}^{(j)}\right\}_{(i, j) \in \Omega}$ of problem (4.1), (4.2), (4.3), and (4.4) satisfies inequality (3.5). 
Remarks 4.2. Comparing Theorem 4.1 with [10, Theorems 1 and 2], we point out that the hypotheses of Theorem 4.1 can be checked more easily. In this paper, we have used a different approach. Our results do not overlap with those from $[9,10]$. Other related works can be found in [1, pages 237-245].

\section{Acknowledgments}

This research was supported by Fondecyt Chile (Grant 1.030.460) and by the Kamea Fund (Israel).

\section{References}

[1] R. P. Agarwal, Difference Equations and Inequalities. Theory, Methods, and Applications, Monographs and Textbooks in Pure and Applied Mathematics, vol. 155, Marcel Dekker, New York, 1992.

[2] B. F. Bylov, R. È. Vinograd, D. M. Grobman, and V. V. Nemyckiı̆, Theory of Ljapunov Exponents and Its Application to Problems of Stability, Izdat. Nauka, Moscow, 1966 (Russian).

[3] M. I. Gil', Norm Estimations for Operator-Valued Functions and Applications, Monographs and Textbooks in Pure and Applied Mathematics, vol. 192, Marcel Dekker, New York, 1995.

[4] Stability of Finite- and Infinite-Dimensional Systems, The Kluwer International Series in Engineering and Computer Science, vol. 455, Kluwer Academic Publishers, Massachusetts, 1998.

[5] M. I. Gil' and R. Medina, The freezing method for linear difference equations, J. Difference Equ. Appl. 8 (2002), no. 5, 485-494.

[6] S. P. Gordon, A stability theory for perturbed difference equations, SIAM J. Control 10 (1972), 671-678.

[7] N. A. Izobov, Linear systems of ordinary differential equations, Itogi Nauki Tekh. Ser. Mat. Anal. 12 (1974), 71-146.

[8] J. P. LaSalle, The Stability of Dynamical Systems, Regional Conference Series in Applied Mathematics, SIAM, Pennsylvania, 1976.

[9] Y.-Z. Lin and S. S. Cheng, Stability criteria for two partial difference equations, Comput. Math. Appl. 32 (1996), no. 7, 87-103.

[10] R. Medina and S. S. Cheng, The asymptotic behavior of the solutions of a discrete reactiondiffusion equation, Comput. Math. Appl. 43 (2002), no. 8-9, 917-925.

[11] J. M. Ortega, Stability of difference equations and convergence of iterative processes, SIAM J. Numer. Anal. 10 (1973), no. 2, 268-282.

Rigoberto Medina: Departmento de Ciencias Exactas, Universidad de Los Lagos, Casilla 933, Osorno, Chile

E-mail address: rmedina@ulagos.cl

M. I. Gil': Department of Mathematics, Ben-Gurion University of the Negev, P.O. Box 653, Beer-Sheva 84105, Israel

E-mail address: gilmi@cs.bgu.ac.il 


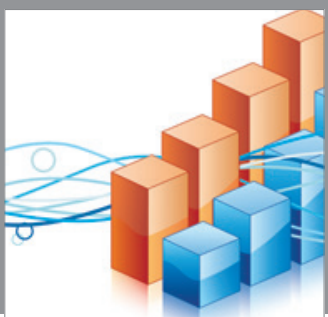

Advances in

Operations Research

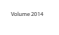

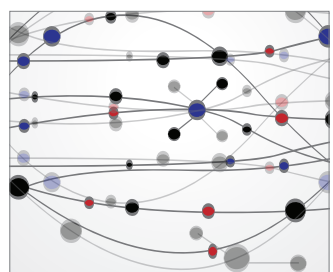

\section{The Scientific} World Journal
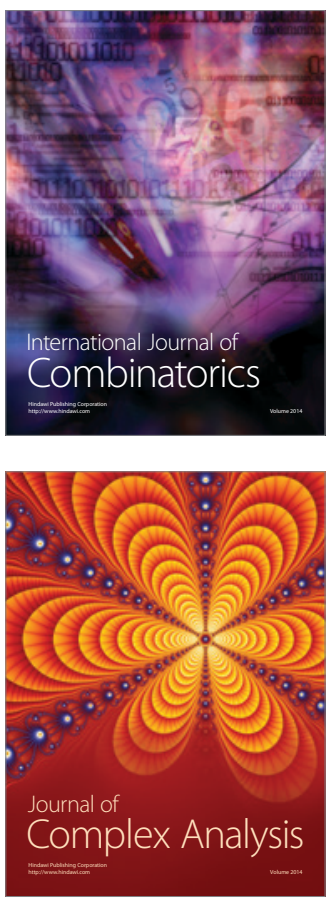

International Journal of

Mathematics and

Mathematical

Sciences
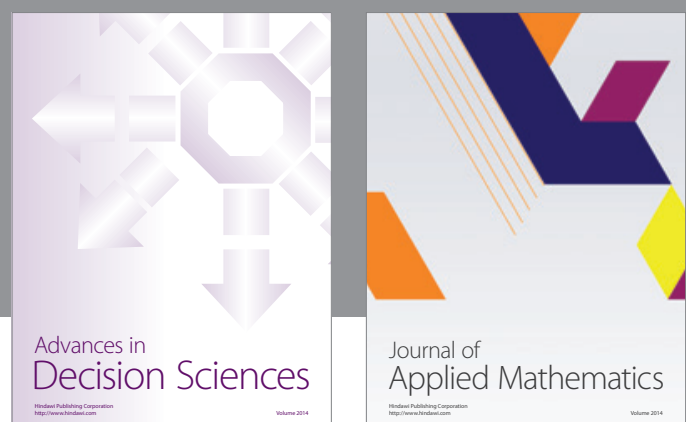

Journal of

Applied Mathematics
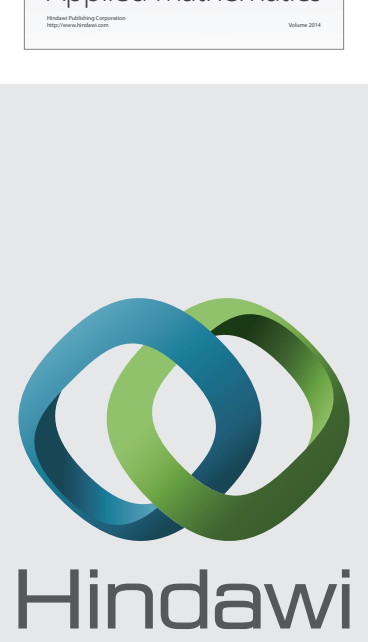

Submit your manuscripts at http://www.hindawi.com
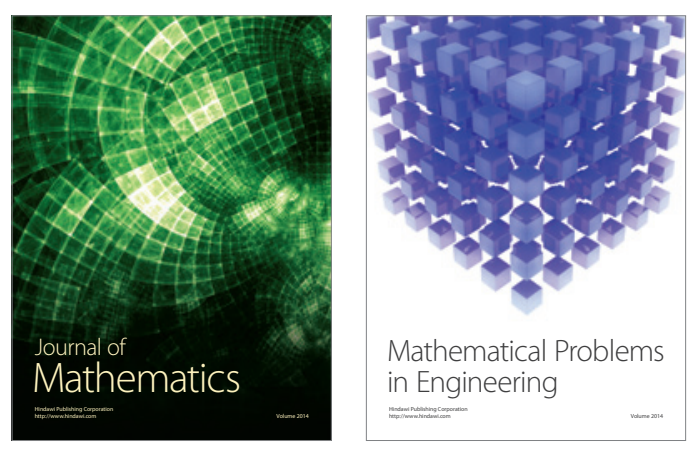

Mathematical Problems in Engineering
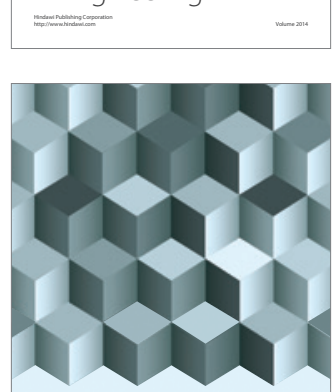

Journal of

Function Spaces
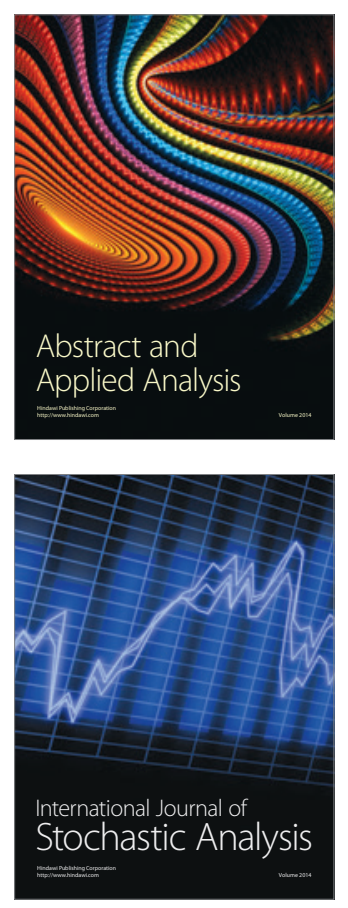

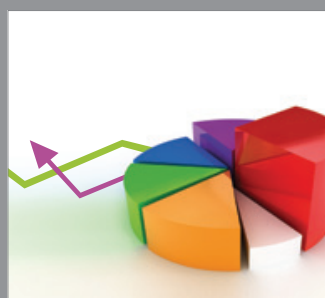

ournal of

Probability and Statistics

Promensencen
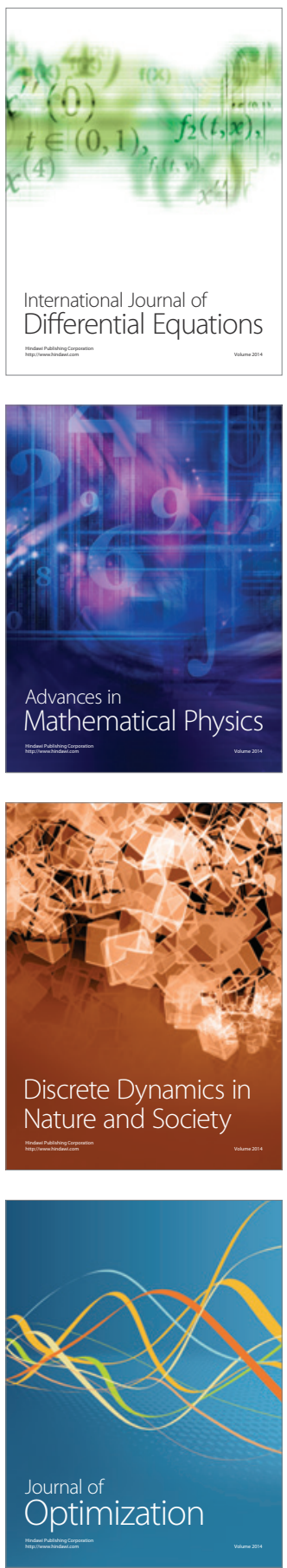